Manuela Tondelli*, Roberta Bedin, Annalisa Chiari, Maria Angela Molinari, Guendalina Bonifacio, Nicoletta Lelli, Tommaso Trenti and Paolo Nichelli

\title{
Role of cerebrospinal fluid biomarkers to predict conversion to dementia in patients with mild cognitive impairment: a clinical cohort study
}

DOI 10.1515/cclm-2014-0414

Received April 15, 2014; accepted August 24, 2014

\section{Abstract}

Background: Cerebrospinal fluid (CSF) levels assessment of $\mathrm{A} \beta_{1-42}$ and Tau proteins may be accurate diagnostic biomarkers for the differentiation of preclinical Alzheimer's disease (AD) from age-associated memory impairment, depression and other forms of dementia in patients with mild cognitive impairment (MCI). The aim of our study was to explore the utility of CSF biomarkers in combination with common cognitive markers as predictors for the risk of $\mathrm{AD}$ development, and other forms of dementia, and the time to conversion in community patients with MCI.

Methods: A group of $71 \mathrm{MCI}$ patients underwent neurological assessment, extended neuropsychological evaluation, routine blood tests, ApoE determination, and lumbar puncture to dose t-tau, $\mathrm{p}$-tau $\mathrm{t}_{181}, \mathrm{~A} \beta_{1-42^{2}}$. We investigated baseline CSF and neuropsychological biomarker patterns according to groups stratified with later diagnoses of $\mathrm{AD}$ conversion (MCI-AD), other dementia (MCI-NAD) conversion, or clinical stability (sMCI).

Results: Baseline $A \beta_{1-42}$ CSF levels were significantly lower in MCI-AD patients compared to both SMCI and MCI-NAD. Additionally, $\mathrm{p}$-tau ${ }_{181}$ was higher in the MCI-AD group compared to SMCI. The MCI-AD subgroup analysis confirmed the role of $A \beta_{1-42}$ in its predictive role of time to conversion: rapid converters had lower $A \beta_{1-42}$ levels compared to

*Corresponding author: Manuela Tondelli, MD, PhD, Biomedical, Metabolic, and Neural Sciences Department, University of Modena and Reggio Emilia, S. Agostino-Estense Hospital, Via Giardini 1355, Baggiovara, Modena, Italy, Phone: +39 059 3961677,

E-mail: manuela.tondelli@unimore.it

Roberta Bedin, Annalisa Chiari, Maria Angela Molinari, Guendalina Bonifacio and Paolo Nichelli: Biomedical, Metabolic, and Neural Sciences Department, University of Modena and Reggio Emilia, S. Agostino-Estense Hospital, Modena, Italy

Nicoletta Lelli and Tommaso Trenti: Clinical Pathology-Toxicology,

S. Agostino-Estense Hospital, Modena, Italy slow converters. Logistic regression and survival analysis further supported the key predictive role of baseline $A \beta_{1-42}$ for incipient $\mathrm{AD}$ and dementia-free survival.

Conclusions: Our results confirm the key role of CSF biomarkers in predicting patient conversion from MCI to dementia. The study suggests that CSF biomarkers may also be reliable in a real world clinical setting.

Keywords: Alzheimer's disease; biomarkers; cerebrospinal fluid; mild cognitive impairment.

\section{Introduction}

Alzheimer's disease (AD) is a chronically progressive neurodegenerative disease and the most common form of dementia in the elderly. It is well known that AD pathological processes appear gradually and begin to develop decades before the onset of clinical symptoms, with dementia representing the end stage of many years of accumulation of these pathological changes [1]. In this continuum, mild cognitive impairment (MCI) has been considered as a half way condition between normal cognition and dementia, with an annual rate of progression to $\mathrm{AD}$ of $15 \%$ [2].

However, not all patients exhibiting MCI will develop AD. Therefore, the possibility of identifying individuals carrying incipient $\mathrm{AD}$ in the MCI population has become of utmost importance. Expert consensus groups have recommended possible primary biomarker candidates, including cerebrospinal fluid (CSF) level of amyloid- $\beta$ 1-42 $\left(\mathrm{A} \beta_{1-42}\right)$, total-tau (t-tau), and phosphorylated tau (p-tau), as well as MRI-based assessments of the hippocampus and enthorinal cortex and neuropsychological markers of memory and non-memory cognitive ability [3].

There are multiple advantages associated with identifying predictive prognostic indicators of conversion from $\mathrm{MCI}$ to $\mathrm{AD}$, including the empowerment for patients and their families to prepare for the future, and the appropriate allocation of early treatment with disease modifying drugs 
in clinical trials to patients with biomarker indications of early conversion to AD whilst not exposing patients unlikely of AD conversion to undue risk. Further, the biomarkers may also be advantageous in prognosis, disease progression analysis, treatment development, treatment effect monitoring and the study of disease mechanism.

CSF biomarkers represent a direct measure of cerebral pathological changes occurring in the AD process. Neurofibrillary tangles and amyloid plaques are the principal pathologic features of $\mathrm{AD}$ and it has been suggested that abnormal processing of amyloid precursor protein leads to a cascade of events characterized by abnormal tau aggregations, synaptic dysfunction, cell death, and changes in brain structure [4]. $A \beta_{1-42}$ is a peptide produced from the cleavage of the amyloid precursor protein (APP) from $\alpha$ and $\gamma$ secretase enzymes. Among different types of $A \beta$ peptides, $A \beta_{1-42}$ has been shown to have the higher specificity and sensitivity in detecting incipient $A D . A \beta_{1-42}$ in CSF has been proven to be significantly reduced in subjects with AD compared to age-matched controls, by about $50 \%$ [5]. Tau proteins are also altered; tau becomes hyperphosphylated and dissociates from the microtubules in $\mathrm{AD}$, causing an increase of CSF total and p-tau [6] in $\mathrm{AD}$ subjects.

Several studies have shown that the relative risk of progression from $\mathrm{MCI}$ to $\mathrm{AD}$ is increased in patients with high baseline tau and $\mathrm{p}$-tau, and low $\mathrm{A} \beta_{1-42}$ with high sensitivity and specificity [7-15]. Following progress in research, a working group established by the National Institute of Aging in 2011 promoted a revision of the diagnostic criteria for $\mathrm{AD}$ and $\mathrm{MCI}$, recommending the adoption of CSF biomarkers for the identification of patients with 'MCI due to $\mathrm{AD}$ ' in research setting, i.e., patients at high risk to develop $\mathrm{AD}$ [16].

The potential of these biomarkers under standardized settings has proven to be effective, with a high diagnostic performance in a homogeneous mono-center population [17]. However, standardization of these biomarkers is currently limited and laboratory results often vary [18, 19]. Community studies are therefore required in order to validate the diagnostic accuracy of CSF measures in everyday clinical settings.

In this study, we explored the utility of CSF biomarkers in combination with standard cognitive markers to identify MCI patients at risk of conversion to $\mathrm{AD}$ and other forms of dementia, in a clinical setting over a 2-year period. We evaluated the best parameter (or combinations of them) for predicting conversion to dementia and time to conversion in a general clinical population, transferring the research approach with precise patient selection used in large cohort studies into a real world clinical scenario.

\section{Materials and methods}

\section{Patients}

A group of 71 patients with clinical diagnosis of amnestic MCI (single domain or multiple domain) recruited from 2007 to 2011 at the Memory Clinic, Department of Neurology, New Civil St. AgostinoEstense Hospital, Modena, Italy, were enrolled into the current study. Clinical baseline assessments were performed, including blood tests, electrocardiogram (ECG), lumbar puncture, extended medical history transcription, and neuropsychological evaluation. Neuropsychological assessments included Mini Mental State Examination (MMSE), Babcock story recall test, Rey-Osterrieth Figure-delay recall test, category and letter fluency test, attention matrices, Stroop test, and Frontal Assessment Battery (FAB). CSF levels of $A \beta_{1-42}$, t-tau and p-tau were measured. Subjects were subsequently followed up clinically every 6 months for at least of 24 months (range 24-31 months) and classified according to whether they had developed AD (MCI$\mathrm{AD}$ converters) or other dementias (MCI-NAD) or whether they had remained stable (sMCI). Baseline differences in CSF and neuropsychological biomarkers patterns were investigated, and MCI patients were stratified according to eventual diagnoses of conversion to $\mathrm{AD}$ or other dementia.

Among the patient cohort, 23 remained stable (sMCI), 31 developed $\mathrm{AD}$ (MCI-AD), and 17 developed non-AD dementia (i.e., frontotemporal dementia, vascular dementia, etc.). In addition, according to literature data suggesting that MCI-AD conversion appears to be time dependent, and occurs primarily within the initial 18 months [20], we further classified MCI-AD patients as rapid ( $\mathrm{n}=16, \leq 18$ months) or slow ( $\mathrm{n}=15,>18$ months) converters. Clinical diagnoses of amnestic MCI, probable $\mathrm{AD}$ or other dementias were made according to published criteria [21-25].

\section{CSF sampling}

Lumbar punctures were performed in the morning in fasting patients; all subjects gave written consent to undergo the lumbar puncture. Approximately $7 \mathrm{~mL}$ CSF were collected in sterile polypropylene tubes and transported to the adjacent laboratory within $30 \mathrm{~min}$. CSF was centrifuged for $15 \mathrm{~min}$ at $2700 \mathrm{~g}$ at controlled room temperature and aliquoted in polypropylene storage tubes. To reduce the possibility of surface adsorption, we aliquoted $400 \mu \mathrm{L}$ CSF in $500 \mu \mathrm{L}$ tubes. If not immediately analyzed, storage tubes were frozen at $-80{ }^{\circ} \mathrm{C}$ awaiting testing. CSF A $\beta_{1-42}$, t-tau, and p-tau ${ }_{181}$ were measured with the ELISA method following manufacturer instructions (Innogenetics, Gent, Belgium). All tests were performed in the same laboratory by the same biologist in accordance with recent guidelines [26]. The inter-assay coefficients of variation (CVs) were within $8.6 \%$ for t-tau, $14.1 \%$ for $\mathrm{A} \beta_{1-42}, 10.9 \%$ for $\mathrm{p}-\operatorname{tau}_{181}$.

\section{Analysis of demographical, neuropsychological, and CSF data}

Baseline demographic, neuropsychological, and CSF data were analyzed with SPSS 20.0 and STATA11 software using parametric or non-parametric tests, as appropriate. First, we investigated baseline 
demographic, neuropsychological, and CSF features between groups of MCI patients stratified by later diagnoses of conversion to AD, other dementia or stable MCI. In particular, ANOVA with post-hoc Bonferroni adjustment or Kruskal-Wallis tests followed by MannWhitney tests for pairwise group comparison were used to compare SMCI, MCI-AD and MCI-NAD data. Correlation analyses were calculated using Spearman's or Pearson's coefficient, as appropriate. Binary logistic regression analyses were performed to distinguish MCI-AD from sMCI and MCI-AD from MCI-NAD. The accuracy of these models were assessed by area under the curve (AUC) of the receiver operating characteristic (ROC) curve. A multinomial logistic regression analysis was then performed to explore the prediction accuracy among the three groups of patients considered together in the analysis. A survival analysis (Cox regression model) was performed to obtain significant predictors of conversion to $\mathrm{AD}$ and of time of conversion. Kaplan-Meier survival analysis was used to determine median dementia-free survival time. Finally, the MCI-AD subgroup was further analyzed according to rapid and slow conversion and an independent t-test was used to compare the CSF biomarkers in the two groups.

\section{Results}

At baseline, there were no significant differences between the groups in terms of age, years of education, gender, MMSE, semantic fluency, prose memory, and t-tau. ApoE4 genotype was prevalent in MCI-AD group compared to other groups, but the difference did not reach statistical significance after correction for multiple comparisons.
Significant differences between MCI-AD and MCI-NAD were detected in verbal fluency, in Stroop test, in $A \beta_{1-42}$ level, and in $A \beta_{1-42 \mid}$-tau and $A \beta_{1-421}$ t-tau ratio. In addition, MCI-AD showed a significant decrease of CSF A $\beta_{1-42}$ level and of $A \beta_{1-42 /} p$-tau ratio and a significant increase of $p$-tau compared to sMCI (Table 1).

Correlation analysis in all MCI groups revealed a significant positive correlation between $\mathrm{A} \beta_{1-42}$, Babcock Story recall test $(r=0.491, p=0.001)$ and Rey-Osterrieth Figuredelay recall test $(r=0.387, p=0.042$; Figure 1$)$. The subgroup analysis performed in the three subgroups of MCI patients separately (MCI-AD, SMCI, MCI-NAD) revealed that only in MCI-AD group there was a significant positive correlation between the three measures; no statistically significant correlation was found in SMCI and MCI-NAD. Other cognitive measures, $A \beta_{1-42}$ or other CSF peptides were not correlated.

Logistic regression analyses showed that $A \beta_{1-42}$ was helpful in differentiating MCI converters from SMCI and both $A \beta_{1-42}$ and $p$-tau were helpful in discriminating between MCI-AD and MCI-NAD. The logistic regression considering $\mathrm{MCI}-\mathrm{AD}$ versus $\mathrm{SMCI}$ as diagnostic status and MMSE, ApoE status, t-tau, $p$-tau and $A \beta_{1-42}$ as independent variables showed that only $A \beta_{1-42}$ was helpful in differentiating MCI converters from SMCI $(\beta=-0.014, \mathrm{SE}=0.006$, $\mathrm{p}=0.034$ ). This model accounted for between $53 \%$ and $72 \%$ of the variance in conversion status, with an overall prediction accuracy of $83 \%$. Hosmer and Lemeshow's

Table 1 Baseline demographic, cognitive and CSF characteristics of $\mathrm{MCl}$ samples.

\begin{tabular}{|c|c|c|c|}
\hline Baseline characteristics & $\begin{array}{r}M C I-A D \\
(n=31)\end{array}$ & $\begin{array}{r}\mathrm{MCI}-\mathrm{NAD} \\
(\mathrm{n}=17)\end{array}$ & $\begin{array}{r}\text { sMCl } \\
(n=23)\end{array}$ \\
\hline Gender, $\mathrm{F} / \mathrm{M}$ & $17 / 14$ & $9 / 8$ & $13 / 10$ \\
\hline Age, years, mean (SD) & $66.2( \pm 9.4)$ & $66.4( \pm 7.6)$ & $68.1( \pm 8.3)$ \\
\hline Education, years, mean (SD) & $10.4( \pm 5.7)$ & $9.5( \pm 4.5)$ & $8.8( \pm 6.4)$ \\
\hline ApoE4 carrier, $\mathrm{y} / \mathrm{n}$ & $10 / 21$ & $4 / 13$ & $2 / 21$ \\
\hline MMSE, mean (SD) & $25.1( \pm 3.1)$ & $25.4( \pm 2.2)$ & $26.8( \pm 2.3)$ \\
\hline Semantic fluency, mean (SD) & $31( \pm 1.9)$ & $35( \pm 1.7)$ & $38( \pm 3.6)$ \\
\hline Phonemic fluency, mean (SD) ${ }^{c}$ & $21( \pm 11.1)$ & $19( \pm 13.1)$ & $29( \pm 9.3)$ \\
\hline Babcock story recall, mean (SD) & $5.04( \pm 1.2)$ & $7.9( \pm 5.2)$ & $6.5( \pm 5.7)$ \\
\hline Rey-Osterrieth Figure-delay recall, mean (SD) ${ }^{\mathrm{b}}$ & $6.1( \pm 7.1)$ & $6.9( \pm 5.2)$ & $11.4( \pm 5.3)$ \\
\hline Frontal Assessment Battery, mean (SD) & $12( \pm 5.1)$ & $12( \pm 5.9)$ & $13( \pm 3.8)$ \\
\hline Stroop test, errors, mean (SD) ${ }^{a, c}$ & $1.0( \pm 2.1)$ & $6.0( \pm 7.4)$ & $1.8( \pm 2.8)$ \\
\hline Follow-up, m, mean (SD) & $28.2( \pm 4.5)$ & $25.5( \pm 1.6)$ & $27.3( \pm 2.4)$ \\
\hline Time of conversion in $\mathrm{MCl}-\mathrm{AD}$, months, mean (SD) & $18.23( \pm 7.56)$ & - & - \\
\hline t-tau pg/mL, mean (SD) & $466.5( \pm 292.4)$ & $448.1( \pm 310.6)$ & $361.6( \pm 357.3)$ \\
\hline P181-tau pg/mL, mean (SD) ${ }^{\mathrm{b}}$ & $101.4( \pm 61.5)$ & $69.1( \pm 40.6)$ & $59.6( \pm 24.3)$ \\
\hline$A \beta 1-42 \mathrm{pg} / \mathrm{mL}$, mean $(\mathrm{SD})^{\mathrm{a}, \mathrm{b}}$ & $442.4( \pm 114.6)$ & $674.5( \pm 303.7)$ & $869.9( \pm 335.4)$ \\
\hline$A \beta 1-42 /$ t-tau (ratio) ${ }^{\mathrm{a}}$ & $1.5( \pm 0.9)$ & $3.8( \pm 2.4)$ & $2.4( \pm 2.9)$ \\
\hline$A \beta 1-42 / p-\operatorname{tau}(\text { ratio) })^{a, b}$ & $7.5( \pm 4.1)$ & $13.8( \pm 10.08)$ & $15.4( \pm 10.3)$ \\
\hline
\end{tabular}

aThere is a significant difference between MCI-AD and MCI-NAD $(p<0.05)$; ba significant difference between $M C I-A D$ and $s M C I(p<0.05)$; ca significant difference between $\mathrm{SMCl}$ and $\mathrm{MCI}-\mathrm{NAD}(\mathrm{p}<0.05)$. 

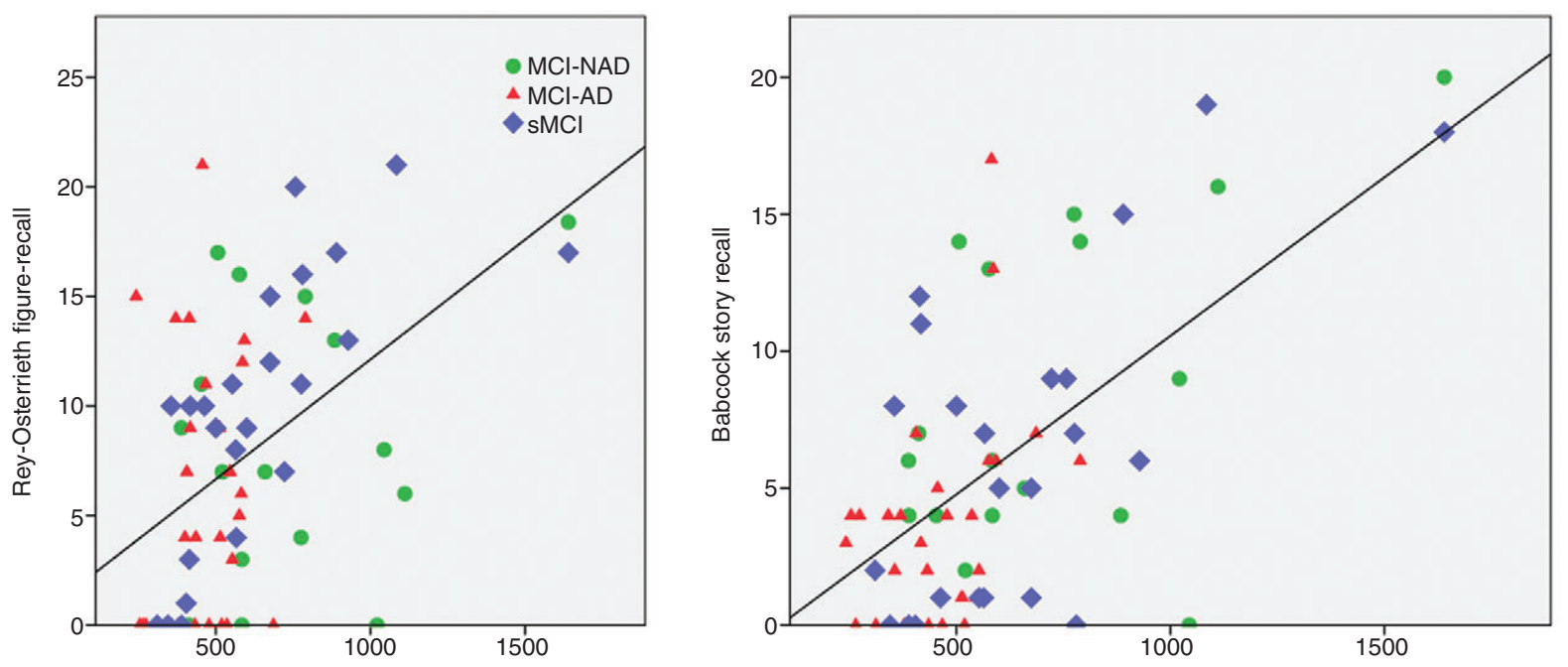

Figure 1 Correlation analyses between memory performances (visuo-spatial and verbal) and $A \beta_{1-42} \mathrm{CSF}$ level (pg/mL). Different symbols and colors indicate different diagnostic groups.

test indicated a high p-value, confirming the efficiency of the model. Sensitivity and specificity of this model were $95.24 \%$ and $80 \%$, respectively (AUC $=0.97$ Figure $2 \mathrm{~B}$, top), the positive predictive value (PPV) was $95.24 \%$ and the negative predictive value (NPV) was $80 \%$.

The logistic regression considering MCI-AD versus MCI-NAD as diagnostic status and the MMSE, ApoE status, $\mathrm{t}$-tau, $\mathrm{p}$-tau and $A \beta 1-42$ as independent variables showed that both $\mathrm{A} \beta_{1-42}(\beta=-0.007, \mathrm{SE}=0.002, \mathrm{p}=0.014)$ and $\mathrm{p}$-tau $(\beta=0.07, \mathrm{SE}=0.032, \mathrm{p}=0.024)$ were helpful in discriminating between MCI-AD and MCI-NAD (Figure 2A). This model accounted for between $37 \%$ and $52 \%$ of the variance in conversion status, with an overall prediction accuracy of $84 \%$ for non-converters. Hosmer and Lemeshow's test indicated a high p-value, confirming the efficiency of the model. The sensitivity and specificity of this model were $85.71 \%$ and $77.27 \%$, respectively (AUC $=0.87$ Figure $2 \mathrm{~B}$, bottom), the PPV was $78.26 \%$ and the NPV $85 \%$.

The logistic regression considering MCI-AD versus MCI-NAD plus sMCI as diagnostic status (i.e., conversion to $\mathrm{AD}$ vs. all other diagnostic status, both stable MCI and MCI converted to other dementia) and MMSE, ApoE status, t-tau, $p$-tau and $A \beta 1-42$ as independent variables showed that only $A \beta_{1-42}(\beta=-0.004, \mathrm{SE}=0.002, \mathrm{p}=0.044)$ was helpful in discriminating MCI patients destined to convert to AD. This model accounted for between 14\% and $19 \%$ of the variance in conversion status, with an overall prediction accuracy of $61 \%$. Again efficacy was confirmed with a high p-value indicated from Hosmer and Lemeshow's test. The sensitivity and specificity of this model were $90.91 \%$ and $41.18 \%$, respectively $(\mathrm{AUC}=0.62)$, the
PPV was $66.67 \%$ and the NPV $77.78 \%$. Due to the heterogeneous nature of the groups of subjects in this model, the specificity and the prediction accuracy significantly decreased compared to previous models. The use of $\mathrm{A} \beta_{1-42 /} \mathrm{p}$-tau and $\mathrm{A} \beta_{1-42 /} \mathrm{t}$-tau ratio in these analyses did not improve predictability.

Finally, the multinomial logistic regression considering MCI-AD versus SMCI versus MCI-NAD as diagnostic status and MMSE, ApoE status, t-tau, p-tau and $A \beta_{1-42}$ as independent variables showed that $A \beta_{1-42}$ was helpful to discriminate between $\mathrm{AD}-\mathrm{MCI}$ and $\mathrm{SMCI}$ (relative risk $=1.01$, $\mathrm{p}=0.019$ ), whereas all CSF biomarkers were helpful to differentiate $\mathrm{AD}-\mathrm{MCI}$ from MCI-NAD (relative risk $\mathrm{A} \beta_{1-42}=1.01$, $\mathrm{p}=0.023$; relative risk $\mathrm{t}$-tau $=1.02, \mathrm{p}=0.031$; relative risk $\mathrm{p}$-tau $=0.87, \mathrm{p}=0.035)$; in this analysis, the MCI-AD group was used as the baseline comparison group. This model accounted for between $61 \%$ and $71 \%$ of the variance in conversion status, with an overall prediction accuracy of $76.7 \%$, which was greater than the proportional by chance accuracy criteria of 55.6, suggesting that the model was useful.

Survival analysis performed with time to AD conversion as a failure covariate showed that MCI patients with higher value of CSF A $\beta_{1-42}$ had a significantly lower risk of AD conversion than individuals with a lower value [hazard ratio $(H R)=0.05 ; \beta=-0.006]$. The presence of a lower CSF $A \beta_{1-42}$ value was associated with a shorter median dementia-free survival (14 months; Figure 3). The cut-off value of $500 \mathrm{pg} / \mathrm{mL}$ for $\mathrm{A} \beta_{1-42}$ was adopted to distinguish between low (i.e., pathological) and high (i.e., normal) CSF levels, as suggested by Sjogren et al. [27]. 

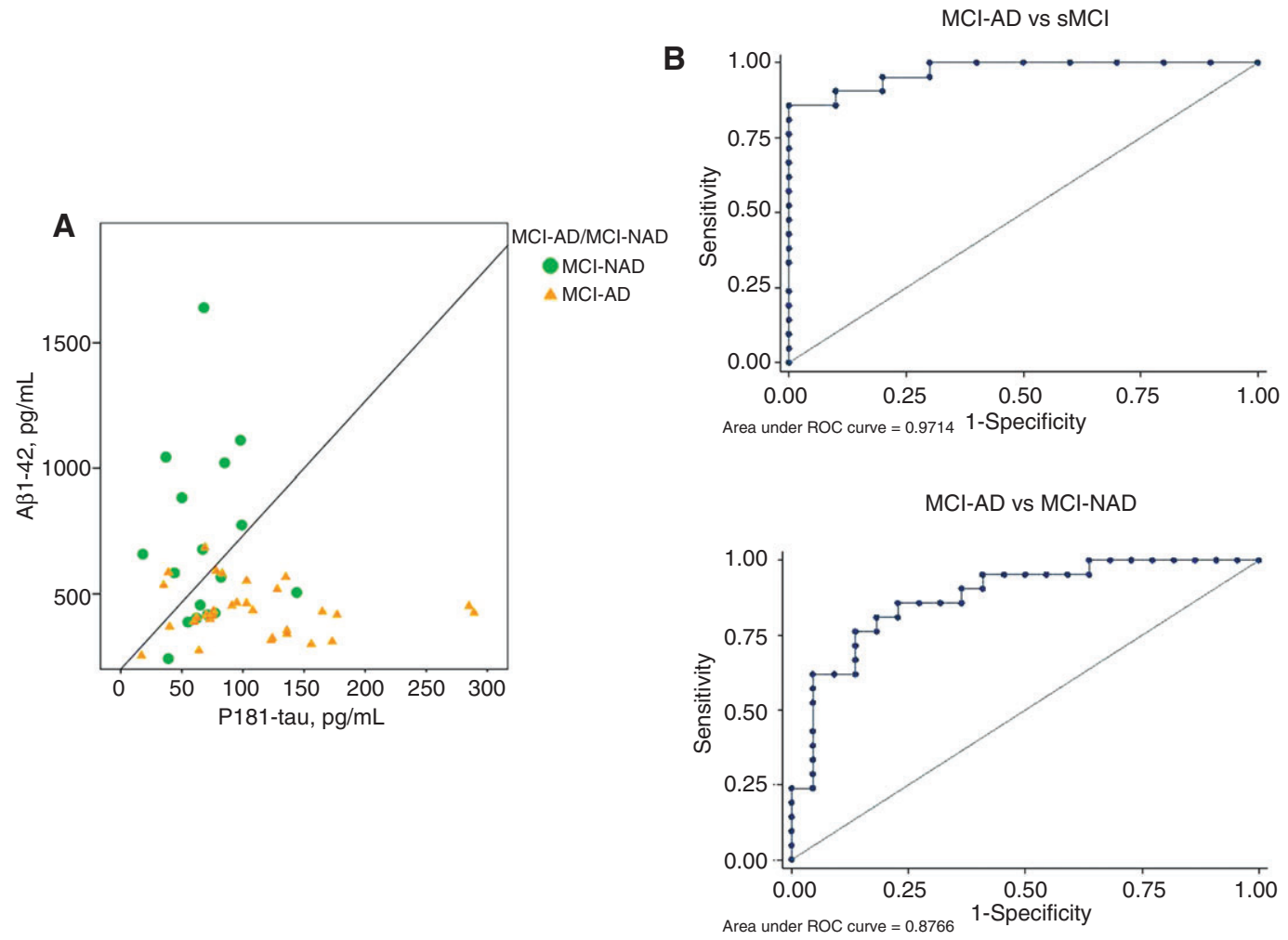

Figure 2 Logistic regression results.

(A) Scatter plot of CSF biomarkers according to the best logistic regression model for MCI-AD compared to MCI-NAD. (B) On the top, ROC curves for the comparison of $\mathrm{MCl}-\mathrm{AD}$ versus SMCl; on the bottom, ROC curves for the comparison of MCI-AD versus MCI-NAD.

In addition, the subgroup analysis between slow and rapid converters confirmed the role of $A \beta_{1-42}$ in distinguishing between the two groups: rapid converters had lower $\mathrm{A} \beta_{1-42}$ baseline levels as compared to slow converters $(\mathrm{p}<0.05$; Figure 4).

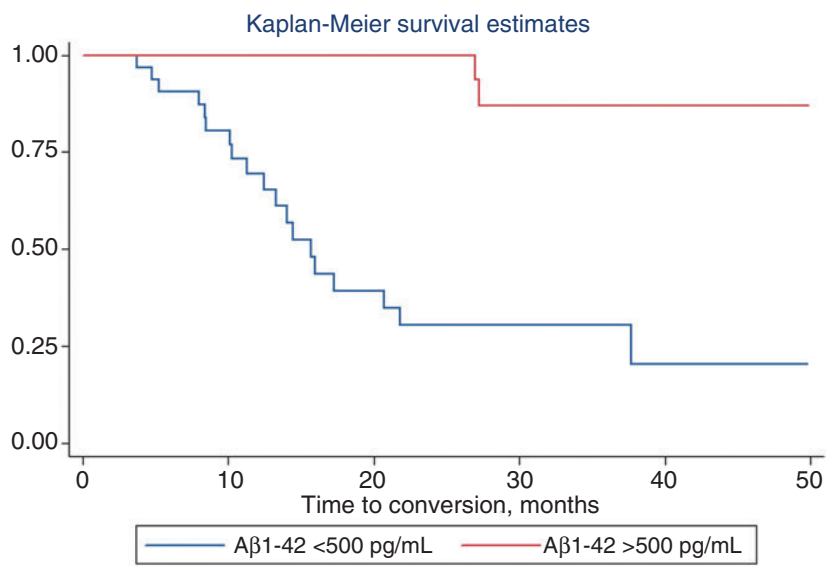

Figure 3 Survival analysis performed with time of conversion to $A D$ as failure covariate in subgroups of $\mathrm{MCl}$ patients classified by $\mathrm{A} \beta_{1-42}$ CSF level. The cut-off value of $500 \mathrm{pg} / \mathrm{mL}$ was adopted according to literature data.

\section{Discussion}

MCI is a heterogeneous disorder. MCI patients may progress to $\mathrm{AD}$, to other dementing illnesses, (including vascular dementia, fronto-temporal dementia and Lewy body dementia), or remain relatively stable and decline cognitively as in normal aging. Therefore, identifying biomarkers of incipient AD before symptoms of dementia are established has many clinical advantages. Among all biological, cognitive, and neuroimaging markers, it has been demonstrated that the combined pattern of low levels of $A \beta_{1-42}$ together with high levels of t-tau and p-tau in CSF may accurately differentiate MCI patients with incipient $\mathrm{AD}$ from those with stable MCI [7].

The aim of the current study was to identify baseline levels of classic CSF biomarkers and assess their powers, either individually or in combination, in predicting conversion to $\mathrm{AD}$ or other dementias. Patients were monitored for at least of 24 months. Patients were then classified according to the development of AD (MCI-AD), other dementia (MCI-NAD) and those who remained cognitively stable (sMCI). 

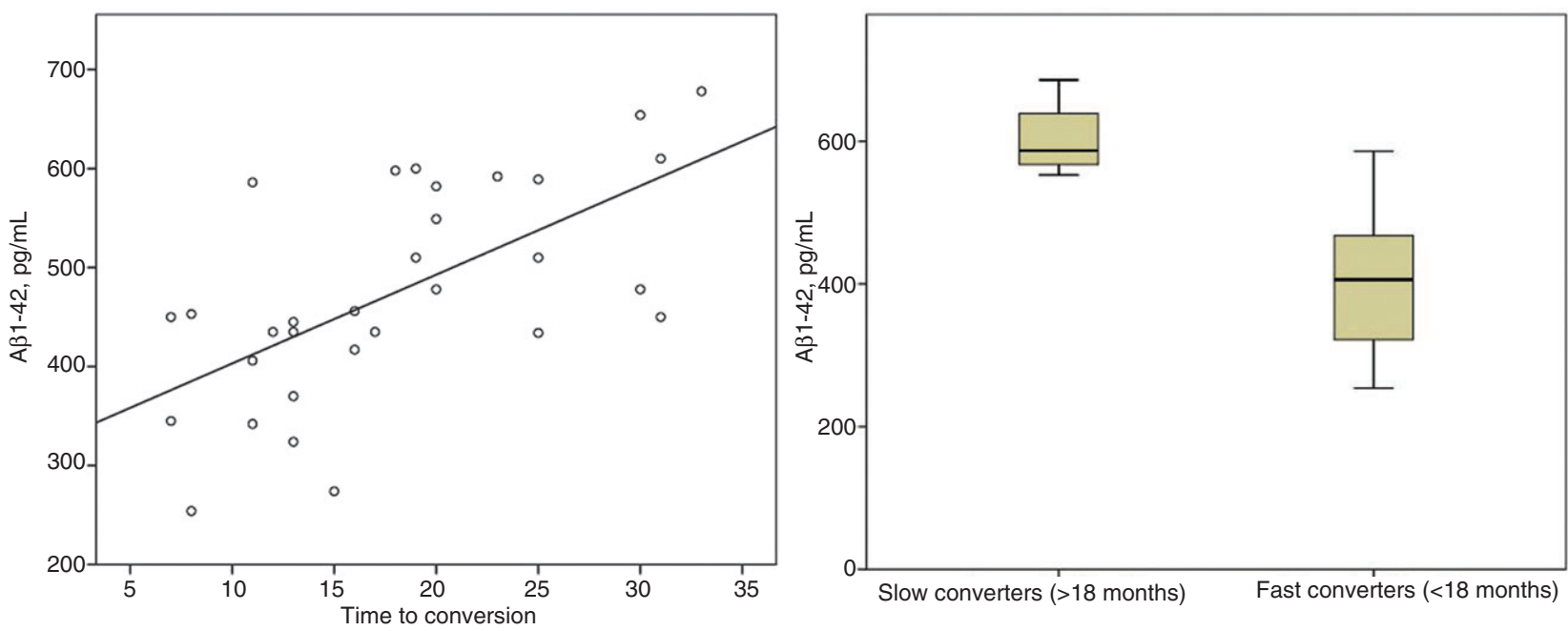

Figure 4 On the left, dementia-free survival time (months) plotted against $A \beta_{1-42}$ CSF values in MCI-AD group. On the right, subgroup analysis between slow ( $>18$ months) and fast $(<18$ months) converters, $p<0.05$.

Retrospective analysis of baseline levels identified $A \beta_{1-42}$ as an important independent biomarker for differentiating MCI-AD patients, and as an indicator of the time to conversion. A significant reduction of $A \beta_{1-42}$ in MCI-AD compared to both MCI-NAD and SMCI, and a significantly lower level of $A \beta_{1-42}$ in rapid converters (i.e., conversion $\leq 18$ months) compared to slow converters (i.e., conversion $>18$ months), were found. Additionally, a significant increase of $\mathrm{p}$-tau in MCI-AD compared to sMCI was evidenced, and t-tau levels were lower in the sMCI group compared to MCI-AD and MCI-NAD groups.

Prognostic factors and biomarkers of time of conversion are not well recognized in literature. Some studies showed an association between time of conversion and injury markers, such as CSF p-tau and t-tau [28, 29], whereas other studies did not find any association [30] or found association with amyloid markers [31]. In our study, $\mathrm{A} \beta_{1-42}$ CSF value was found to be a predictor not only of conversion, but also of time of conversion, whereas we did not find this association with neurodegeneration markers (i.e., t-tau and p-tau). In a study by Van Rossum and colleagues [28], the authors found that injury markers but not amyloid markers were associated with faster decline from $\mathrm{MCI}$ to $\mathrm{AD}$, but MCI subjects who went on to convert to $\mathrm{AD}$ had already at baseline lower MMSE scores compared to subjects who did not progress to AD dementia, suggesting that these patients were already at baseline in a more advanced stage of the disease, where it is well known that markers of neurodegeneration are more useful in predicting prognosis than amyloid markers. On the contrary, in our study, no significant differences between the groups were found in CSF tau level and MMSE at baseline, suggesting that our MCI subjects did not differ in terms of neurodegenerative process stage at the first visit. Maybe, these differences in patient's characteristics and selection criteria may account for the discrepancies emerged in our study compared to previous one in terms of time of conversion. In addition, we cannot rule out the presence of inter-laboratory differences related to the variability of CSF biomarkers assays.

Statistical analyses of different combinations of biomarkers converged to emphasize the central role of $A \beta_{1-42}$ in differentiating the incipient $A D$ type. $A \beta_{1-42}$ and p-tau were identified through regression and survival models as the best predictors for discriminating incipient AD in MCI population, in line with previous studies [7, 9, 10, 15, 30, 32]. Additionally, $A \beta_{1-42}$ was found to be significantly correlated with memory performances on cognitive tests. This correlation was limited to the MCI-AD group, confirming the early and specific involvement of memory in preclinical phases of the disease [33]. Despite this correlation, common cognitive performances were not found to be strong predictors of AD conversion in our models. These results suggest that CSF biomarkers provide predictive information which cannot be obtained from clinical and neuropsychological assessment only.

Both binary and multinomial logistic regression showed high specificity and sensitivity in detecting AD-MCI among SMCI and MCI-NAD separately, whereas the level of accuracy decreased for the discrimination of incipient $\mathrm{AD}$ among more mixed group of patients. As a matter of fact, we cannot exclude the presence of Alzheimer's histopathology in patients clinically classified as SMCI or MIC-NAD, in line with literature data that reported 
high sensitivity but low specificity for the AD-like CSF profile [34]. Unfortunately, we did not have histopathological examination to support our results. Maybe a longer follow-up could have helped to assess whether patients with SMCI eventually progress to AD-type dementia.

This study is limited by a relative small sample size compared to large research cohort studies. However, the study offers insight into the investigation of the role of biomarkers in a real 'clinical community setting'. The advantages associated with the clinical community setting are mainly associated with patient selection. Large research cohort studies enroll carefully selected MCI participants with extensively documented memory impairment, and often exclude those with impairment arising from other potential causes, resulting in an MCI population which doesn't reflect the general clinical population. The current study is based on a general clinical patient cohort and confirms the utility of biomarkers in detecting $\mathrm{AD}$ neuropathological changes in MCI patients. Indeed, we included a heterogeneous group of MCI patients in the testing of specificity and sensitivity of biomarkers in discriminating different neuropathological conditions at a preclinical stage. Additional research is required to validate the application of CSF biomarkers in clinical settings, and procedures for acquisition and analysis of samples need to be established for broad scale application.

Our results suggest that the application of both clinical criteria and CSF biomarkers may reliably predict the conversion of $\mathrm{MCI}$ to $\mathrm{AD}$, and the time to conversion, even in a routine diagnostic work-up.

Acknowledgments: The authors would like to thank Johanna Chester for her critical revision and editorial assistance.

Author contributions: All the authors have accepted responsibility for the entire content of this submitted manuscript and approved submission.

Financial support: None declared.

Employment or leadership: None declared.

Honorarium: None declared.

Competing interests: None declared.

\section{References}

1. Jack CR, Jr., Knopman DS, Jagust WJ, Petersen RC, Weiner MW, Aisen PS, et al. Tracking pathophysiological processes in Alzheimer's disease: an updated hypothetical model of dynamic biomarkers. Lancet Neurol 2013;12:207-16.
2. Petersen RC, Roberts RO, Knopman DS, Boeve BF, Geda YE, Ivnik RJ, et al. Mild cognitive impairment: ten years later. Arch Neurol 2009;66:1447-55.

3. Frisoni GB, Blennow K. Biomarkers for Alzheimer's: the sequel of an original model. Lancet Neurol 2013;12:126-8.

4. Hardy J, Selkoe DJ. The amyloid hypothesis of Alzheimer's disease: progress and problems on the road to therapeutics. Science 2002;297:353-6.

5. Holtzman DM. CSF biomarkers for Alzheimer's disease: current utility and potential future use. Neurobiol Aging 2011;32 (Suppl 1):S4-9.

6. Blennow K, Wallin A, Agren H, Spenger C, Siegfried J, Vanmechelen E. Tau protein in cerebrospinal fluid: a biochemical marker for axonal degeneration in Alzheimer disease? Mol Chem Neuropathol. 1995;26:231-45.

7. Blennow K, Hampel H. CSF markers for incipient Alzheimer's disease. Lancet Neurol 2003;2:605-13.

8. Blennow K, Vanmechelen E. CSF markers for pathogenic processes in Alzheimer's disease: diagnostic implications and use in clinical neurochemistry. Brain Res Bull 2003;61:235-42.

9. Mattsson N. CSF biomarkers in neurodegenerative diseases. Clin Chem Lab Med 2011;49:345-52.

10. Mattsson N, Blennow K, Zetterberg H. CSF biomarkers: pinpointing Alzheimer pathogenesis. Ann NY Acad Sci 2009;1180:28-35.

11. Mattsson N, Insel P, Nosheny R, Zetterberg H, Trojanowski JQ, Shaw LM, et al. CSF protein biomarkers predicting longitudinal reduction of CSF beta-amyloid42 in cognitively healthy elders. Transl Psychiatry 2013;3:e293.

12. Mattsson N, Zetterberg H. Alzheimer's disease and CSF biomarkers: key challenges for broad clinical applications. Biomark Med 2009;3:735-7.

13. Mattsson N, Zetterberg H, Hansson O, Andreasen N, Parnetti L, Jonsson M, et al. CSF biomarkers and incipient Alzheimer disease in patients with mild cognitive impairment. J Am Med Assoc 2009;302:385-93.

14. Parnetti L, Chiasserini D. Role of CSF biomarkers in the diagnosis of prodromal Alzheimer's disease. Biomark Med 2011;5:479-84.

15. Parnetti L, Chiasserini D, Eusebi P, Giannandrea D, Bellomo G, De Carlo C, et al. Performance of abeta1-40, abeta1-42, total tau, and phosphorylated tau as predictors of dementia in a cohort of patients with mild cognitive impairment. J Alzheimers Dis 2012;29:229-38.

16. Albert MS, DeKosky ST, Dickson D, Dubois B, Feldman HH, Fox NC, et al. The diagnosis of mild cognitive impairment due to Alzheimer's disease: recommendations from the National Institute on Aging-Alzheimer's Association workgroups on diagnostic guidelines for Alzheimer's disease. Alzheimers Dement 2011;7:270-9.

17. Johansson P, Mattsson N, Hansson O, Wallin A, Johansson JO, Andreasson U, et al. Cerebrospinal fluid biomarkers for Alzheimer's disease: diagnostic performance in a homogeneous mono-center population. J Alzheimers Dis 2011;24:537-46.

18. Mattsson N, Andreasson U, Persson S, Arai H, Batish SD, Bernardini S, et al. The Alzheimer's Association external quality control program for cerebrospinal fluid biomarkers. Alzheimers Dement 2011;7:386-95 e6.

19. Mattsson N, Andreasson U, Persson S, Carrillo MC, Collins S, Chalbot S, et al. CSF biomarker variability in the Alzheimer's Association quality control program. Alzheimers Dement 2013;9:251-61. 
20. Busse A, Angermeyer MC, Riedel-Heller SG. Progression of mild cognitive impairment to dementia: a challenge to current thinking. Br J Psychiatry 2006;189:399-404.

21. McKhann G, Drachman D, Folstein M, Katzman R, Price D, Stadlan EM. Clinical diagnosis of Alzheimer's disease: report of the NINCDS-ADRDA Work Group under the auspices of Department of Health and Human Services Task Force on Alzheimer's Disease. Neurology 1984;34:939-44.

22. Neary D, Snowden JS, Gustafson L, Passant U, Stuss D, Black S, et al. Frontotemporal lobar degeneration: a consensus on clinical diagnostic criteria. Neurology 1998;51:1546-54.

23. Petersen RC, Smith GE, Waring SC, Ivnik RJ, Tangalos EG, Kokmen E. Mild cognitive impairment: clinical characterization and outcome. Arch Neurol 1999;56:303-8.

24. Rockwood K, Parhad I, Hachinski V, Erkinjuntti T, Rewcastle B, Kertesz A, et al. Diagnosis of vascular dementia: consortium of Canadian Centres for Clinical Cognitive Research concensus statement. Can J Neurol Sci 1994;21:358-64.

25. Winblad B, Palmer K, Kivipelto M, Jelic V, Fratiglioni L, Wahlund LO, et al. Mild cognitive impairment - beyond controversies, towards a consensus: report of the International Working Group on Mild Cognitive Impairment. J Intern Med 2004;256:240-6.

26. Vanderstichele H, Bibl M, Engelborghs S, Le Bastard N, Lewczuk P, Molinuevo JL, et al. Standardization of preanalytical aspects of cerebrospinal fluid biomarker testing for Alzheimer's disease diagnosis: a consensus paper from the Alzheimer's Biomarkers Standardization Initiative. Alzheimers Dement 2012;8:65-73.

27. Sjogren $M$, Vanderstichele $H$, Agren $H$, Zachrisson 0 , Edsbagge $M$, Wikkelso $\mathrm{C}$, et al. Tau and Abeta42 in cerebrospinal fluid from healthy adults 21-93 years of age: establishment of reference values. Clin Chem 2001;47:1776-81.

28. van Rossum IA, Visser PJ, Knol DL, van der Flier WM, Teunissen CE, Barkhof F, et al. Injury markers but not amyloid markers are associated with rapid progression from mild cognitive impairment to dementia in Alzheimer's disease. J Alzheimers Dis 2012;29:319-27.

29. van Rossum IA, Vos SJ, Burns L, Knol DL, Scheltens P, Soininen $\mathrm{H}$, et al. Injury markers predict time to dementia in subjects with $\mathrm{MCl}$ and amyloid pathology. Neurology 2012;79:1809-16.

30. Hansson O, Zetterberg H, Buchhave P, Londos E, Blennow K, Minthon L. Association between CSF biomarkers and incipient Alzheimer's disease in patients with mild cognitive impairment: a follow-up study. Lancet Neurol 2006;5:228-34.

31. Roe CM, Fagan AM, Grant EA, Hassenstab J, Moulder KL, Maue Dreyfus D, et al. Amyloid imaging and CSF biomarkers in predicting cognitive impairment up to 7.5 years later. Neurology 2013;80:1784-91.

32. Rosen C, Hansson O, Blennow K, Zetterberg H. Fluid biomarkers in Alzheimer's disease - current concepts. Mol Neurodegener 2013;8:20.

33. Haldenwanger A, Eling P, Kastrup A, Hildebrandt H. Correlation between cognitive impairment and CSF biomarkers in amnesic $\mathrm{MCl}$, non-amnesic $\mathrm{MCl}$, and Alzheimer's disease. J Alzheimers Dis 2010;22:971-80.

34. De Riva V, Galloni E, Marcon M, Di Dionisio L, Deluca C, Meligrana L, et al. Analysis of combined CSF biomarkers in AD diagnosis. Clin Lab 2014;60:629-34. 